\title{
Fidelity and Fidelity Susceptibility of Pulses in Dispersive Media
}

\author{
Li-Gang Wang ${ }^{1,2}$ and Shi-Jian Gu ${ }^{1}$ \\ ${ }^{1}$ Department of Physics, The Chinese University of Hong Kong, Shatin, N. T., Hong Kong \\ ${ }^{2}$ Department of Physics, Zhejiang University, Hangzhou, 310027, China
}

\begin{abstract}
Motivated by the growing importance of the fidelity and fidelity susceptibility (FS) in quantum critical phenomena, we use these concepts to describe the pulse propagation inside the dispersive media. It is found that there is a dramatic change in the fidelity and the FS of the pulse at a critical propagation distance inside a dispersive medium, and whether such a dramatic change for a light pulse occurs or not strongly depends on both the dispersive strength of the media and the pulse property. We study in detail about the changes of the fidelity and the FS for both a smooth and a truncated Gaussian pulse through the abnormal and normal dispersive media, where the group velocities are well defined. Our results show that both the fidelity and the FS could be very useful to determine whether the pulse is completely distorted or not at the critical distance, therefore it would be very helpful to find the maximal effective propagation region of the pulse's group velocity, in terms of the changes of the pulse's fidelity and FS.

PACS numbers: 42.25.Bs, 03.65.Ca
\end{abstract}




\section{INTRODUCTION}

Recently, to control the pulse propagation inside various media has attracted intensive attention due to potential applications in information processing and communication systems. It is now well known that the group velocity of a light pulse can be controlled by tailoring the dispersive properties of various materials [1, 2]. Many recent experiments [3, 4, 5, 6, 7] have demonstrated that the group velocity can be larger than the speed of light in vacuum, and even become negative in various situations, such as anomalous dispersive media and photonic band-gap materials. It has been discovered also that the group velocity could become extremely slow in normal dispersive media, such as an ultracold atomic gas [8] and solid-state materials [9, 10]. However, due to the effects of materials' dispersion and absorption (or gain), the light pulse's shape always suffers an inevitable distortion, which is directly related to the propagation distance of the pulse inside a given medium. Then one may wonder: is there any limitation on the propagation distance for a light pulse inside the dispersive media? And how to quantitatively determine the maximal effective propagation region for a given light pulse without distorting its original shape? Since it is very difficult to quantitatively measure the degree of the pulse distortion, to the best of our knowledge, the above questions have not been answered.

The concept of fidelity is originally defined in the field of quantum information theory. It measures the similarity between two quantum states [11, 12]. Recently, this concept has been used to characterize quantum phase transitions in various quantum many-body systems [13, 14, 15, 16, 17, 18]. It has been found that a dramatic change of a quantum state should result in a great change in fidelity across a critical point. In prior studies, the quantum fidelity is used to depict the distance between two slightly different states corresponding to two slightly different values of the parameters [14] in the parameter space. Thus fidelity reflects a response of a quantum state to a change of the driving parameter. In order to study the fidelity, one should in principle choose a small distance in the parameter space. This distance is arbitrary and not so important in relevant studies. It was later pointed out that leading term of fidelity, called fidelity susceptibility (FS), plays a central role [19, 20, 21, 22] in the fidelity approaches to quantum phase transitions.

In this paper, we use the fidelity and the FS of a pulse wavepacket and try to answer the above questions in terms of the changes of fidelity and FS. We find that the fidelity and the 
FS are very useful to determine the range of the effective propagation distance for a light pulse almost preserving its original shape before a strong distortion of the pulse wavepacket happens inside dispersive media.

This paper is organized as follows: In Sec. II, we simply present a brief proof on the equivalent description of a light pulse wavepacket in both the temporal and frequency domains. In Sec. III, we define the concepts of the fidelity and the FS for a light pulse propagating through a dispersive medium in the frequency domain. In Sec. IV, we study numerically the changes of the fidelity and the FS of both a smooth and a truncated Gaussian pulses inside the different types of dispersive media. We also analyze the influence of both the dispersive media and the pulse itself on the changes of the fidelity and the FS. Finally, our conclusions are given in Sec. V.

\section{AN EQUIVALENT DESCRIPTION OF A PULSE WAVEPACKET BETWEEN THE TEMPORAL AND FREQUENCY DOMAINS}

It is well-known that, within a linear response theory and a moving time frame $\tau$ ( $\tau=$ $t-z / c$, here $c$ is the light speed in vacuum), the evolution equation of the wavepacket $\Psi(z, \tau)$ for a light pulse propagating inside a dispersive medium can be expressed as [23, 24]

$$
\Psi(z, \tau)=\frac{1}{\sqrt{2 \pi}} \int \Psi\left(0, t_{1}\right) G\left(\tau-t_{1}, z\right) d t_{1},
$$

where $\Psi\left(0, t_{1}\right)$ is the initial wave packet of a light pulse at position $z=0$ in the temporal domain, and $G\left(\tau-t_{1}, z\right)$ is the medium's Green function given by

$$
G\left(\tau-t_{1}, z\right)=\frac{1}{\sqrt{2 \pi}} \int \exp \left[-i \omega\left(\tau-t_{1}\right)\right] \exp \left\{\frac{i \omega[n(\omega)-1+i \kappa(\omega)] z}{c}\right\} d \omega .
$$

Here the real functions $n(\omega)$ and $\kappa(\omega)$ are related to the complex refractive index, satisfying the relation:

$$
n(\omega)+i \kappa(\omega)=\sqrt{\epsilon(\omega)},
$$

where $\epsilon(\omega)$ is the dielectric function of a non-magnetic medium. From Eqs. (11) and (2), we can find the relation:

$$
\psi(z, \omega)=\psi(0, \omega) \exp \left\{\frac{i \omega[n(\omega)-1+i \kappa(\omega)] z}{c}\right\}
$$


in the frequency domain, where $\psi(z, \omega)$ is the spectral wavepacket in the frequency domain, $\Psi(z, \tau)$ an inverse Fourier transformation of the wave packet in the temporal domain. In Eq. (4), the function $\psi(0, \omega)$ is given by

$$
\psi(0, \omega)=\frac{1}{\sqrt{2 \pi}} \int \Psi\left(0, t_{1}\right) \exp \left(i \omega t_{1}\right) d t_{1},
$$

which is actually a transformation for the initial wave packet $\Psi\left(0, t_{1}\right)$ from the temporal domain into the frequency domain. From Eq. (4), we can find that in the moving time frame the spectral wave packet $\psi(z, \omega)=\psi(0, \omega)$ keeps unchanged in the vacuum $[n(\omega)=1$ and $\kappa(\omega)=0]$. This result is in a good agreement with our common sense that the light temporal wavepacket in vacuum is unchanged in the moving time frame. Therefore, for the description of a light wave packet, the expression $\Psi(z, \tau)$ in the temporal domain is totally equivalent to the expression $\psi(z, \omega)$ in the frequency domain.

\section{FIDELITY AND FIDELITY SUSCEPTIBILITY IN DISPERSIVE MEDIA}

Now we would like to use the wavepacket in the frequency domain to define the fidelity and the FS of a light pulse when it propagates inside a dispersive medium. Generally, in a dispersive medium $\left[n_{r}(\omega) \neq 1\right.$ and $\left.\kappa(\omega) \neq 0\right]$, the pulse spectral wavepacket changes due to the medium's dispersion and absorption (or gain) as it propagates over a distance $z$. Therefore the fidelity of a light pulse wave packet must change as it propagates through a medium.

We consider the propagation of a light pulse passing through a non-magnetic dispersive medium with its dielectric function

$$
\epsilon(\omega)=1+\chi_{M}(\omega)
$$

where $\chi_{M}(\omega)$ is the linear susceptibility of the dispersive medium, such as the two-level [25], three-level [4, 26], or multilevel atomic gas media [27, 28, 29]. In the frequency domain, the wave packet of a light pulse at position $z$ is $\psi(z, \omega)$. After it passes through a medium with a length $\delta z$, the pulse spectral wave packet becomes $\psi(z+\delta z, \omega)$. Following the definition in Ref. [14], the fidelity is defined as the modul of the overlap of two quantum states. In our cases of classical wavepacket, the fidelity can be also defined as the overlap between two 
slightly different wavepackets $\psi(z, \omega)$ and $\psi(z+\delta z, \omega)$ in the frequency domain, that is,

$$
F(z, \delta z)=\frac{1}{N(z, \delta z)}\left|\int \psi(z, \omega) \cdot \psi^{*}(z+\delta z, \omega) d \omega\right|
$$

where $N(z, \delta z)$ is a real normalized function, which is given by

$$
N(z, \delta z)=\left[\int|\psi(z, \omega)|^{2} d \omega \int|\psi(z+\delta z, \omega)|^{2} d \omega\right]^{1 / 2} .
$$

Now the propagation distance $z$ becomes a driving parameter. Combining with Eq. (4), we can see from Eq. (7) that the fidelity $F$ is exactly equal to one in the vacuum $\left[n_{r}(\omega)=1\right.$ and $\kappa(\omega)=0]$. This indicates that the light pulse does not change in vacuum. At the same time, from Eq. (17), we can see that as $\delta z$ approaches zero, $F$ still goes to one even if the pulse propagates inside the dispersive medium.

In general, when a pulse propagates inside the dispersive medium, $F$ is a well-defined monotonical and decreasing function with respect to $\delta z$. Therefore, we can expand Eq. (7) by Taylor's expansion, (for example, see Refs. [20, 22])

$$
\begin{aligned}
\left.F(z, \delta z)\right|_{\delta z \rightarrow 0}= & F(z, \delta z=0)+\left.\frac{\partial F(z, \delta z)}{\partial \delta z}\right|_{\delta z=0} \delta z \\
& +\left.\frac{1}{2} \frac{\partial^{2} F(z, \delta z)}{\partial^{2} \delta z}\right|_{\delta z=0}(\delta z)^{2}+O\left((\delta z)^{3}\right), \\
= & 1-\left[-\left.\frac{1}{2} \frac{\partial^{2} F(z, \delta z)}{\partial^{2} \delta z}\right|_{\delta z=0}\right](\delta z)^{2}+O\left((\delta z)^{3}\right) .
\end{aligned}
$$

We can analytically prove the first derivative in Eq. (9) to be zero given the parameter $\delta z \sim 0$, therefore the most relevant term in determining the fidelity of a light pulse is its second derivative. According to the response theory, the coefficient term before $(\delta z)^{2}$ actually defines a response of fidelity to a small change in the propagation distance. Following with Ref. [20], we can introduce the FS for a light pulse as follows,

$$
\chi_{F}(z)=-\left.\frac{2 \ln F}{(\delta z)^{2}}\right|_{\delta z=0} .
$$

With Eq. (7), the FS in a dispersive medium can be expressed as

$$
\chi_{F}(z)=-\frac{M^{2}(z)}{P^{2}(z)}+\frac{2 M(z) \operatorname{Re}[Q(z)]}{P^{2}(z)}+\frac{U(z)}{2 P(z)}-\frac{\operatorname{Re}[X(z)]}{P(z)}-\frac{|Q(z)|^{2}}{P^{2}(z)},
$$

where the functions $P(z), M(z), Q(z), U(z)$, and $X(z)$ are defined as follows

$$
P(z) \equiv N(z, \delta z=0)
$$




$$
\begin{gathered}
M(z) \equiv-2 \int \frac{\omega}{c} \kappa(\omega)|\psi(z, \omega)|^{2} d \omega \\
Q(z) \equiv \int\left\{\frac{i \omega}{c}[n(\omega)-1]-\frac{\omega}{c} \kappa(\omega)\right\}|\psi(z, \omega)|^{2} d \omega \\
U(z)=4 \int \frac{\omega^{2}}{c^{2}} \kappa^{2}(\omega)|\psi(z, \omega)|^{2} d \omega \\
X(z) \equiv \int\left\{\frac{i \omega}{c}[n(\omega)-1]-\frac{\omega}{c} \kappa(\omega)\right\}^{2}|\psi(z, \omega)|^{2} d \omega .
\end{gathered}
$$

From Eq. (13), we can readily obtain the change of the FS in the dispersive medium. For the vacuum, since $n(\omega)-1=0$ and $\kappa(\omega)=0$ for all $\omega$, one can easily verify that the value of $\chi_{F}(z)$ is always equal to zero, which means that the status of the light pulse does not change in the vacuum. This conclusion is in agreement with that from Eq. (17). In the following discussion, we will show that the change of $\chi_{F}(z)$ indicates the different response properties of the different dispersive media for a light pulse.

\section{NUMERICAL RESULTS AND DISCUSSIONS}

In this section, we would like to discuss the changes of the fidelity and the FS of a light pulse in two types of non-magnetic dispersive media, such as double-gain anomalous dispersive media and double-absorptive normal dispersive media, where the group velocity has been well defined and could be superluminal or subluminal. The linear susceptibility of the media in our cases is a form of double Lorentz oscillators [4],

$$
\chi_{M}(\omega)=\frac{M}{\omega-\omega_{0}-\Delta+i \gamma}+\frac{M}{\omega-\omega_{0}+\Delta+i \gamma},
$$

where $M$ is proportional to the strength of the two oscillators, $\Delta$ the frequency detuning, $\gamma$ the damping rate of the two oscillators, and $\omega_{0}$ the pulse carried frequency. Such a linear susceptibility $\chi_{M}(\omega)$ may describe a three-level atomic system with two closely placed Raman gain peaks [4]. Near $\omega_{0}$, when $M<0$, the medium is an absorptive normal dispersive medium, and when $M>0$, it becomes a gain abnormal dispersive medium. In the following two subsections, we will show that the changes of the fidelity and the FS are strongly affected by different types of dispersive media and the property of the pulse itself. From the fidelity and the FS of the pulse inside the dispersive medium, one can determine the effective propagation distance of a pulse. 


\section{A. A smooth Gaussian pulse}

First, let us consider the propagation of a smooth Gaussian pulse passing through a dispersive media. For a smooth Gaussian pulse, its wavepacket in the time domain is assumed to be in the Gaussian form

$$
\Psi\left(0, t_{1}\right)=A_{0} \exp \left[-\frac{t_{1}^{2}}{2 T_{0}^{2}}\right] \exp \left[-i \omega_{0} t_{1}\right]
$$

at the incident end $z=0$, where $A_{0}$ is a constant, $T_{0}$ is the temporal half-width of the Gaussian pulse, and $\omega_{0}$ is the carried frequency. Its corresponding pulse spectral wavepacket in the frequency domain could be readily obtained as follows [23]

$$
\psi(0, \omega)=\frac{T_{0} A_{0}}{2 \sqrt{\pi}} \exp \left[-\frac{T_{0}^{2}\left(\omega-\omega_{0}\right)^{2}}{2}\right] .
$$

Using Eq. (4), we can find the pulse spectral wavepacket at any position inside dispersive media. Then using Eq. (17), we can obtain the fidelity $F(0, z)$ between the initial pulse and the pulse at a certain distance. At the same time, using Eq. (13), we can find the change of the FS for optical pulses inside dispersive media. Without loss of generality, we take $\omega_{0} / 2 \pi=3.5 \times 10^{14} \mathrm{~Hz}$, and pulse temporal width $T_{0}=1.2 \mu \mathrm{s}$ in the next numerical calculations.

Figure 1 shows the changes of the fidelity $F(0, z)$ and the FS $\chi_{F}(z)$ for a smooth Gaussian pulse propagating through a double-gain anomalous dispersive medium with $M>0$ in Eq. (19). From Figs. 1(a) and 1(c), the fidelity is a monotonical, decreasing function. Initially the fidelity smoothly decreases with respect to the propagation distance $z$, and after a critical propagation distance $z_{c}$ we find that the fidelity suddenly decreases very quickly. Correspondingly, close to the sudden decrease of the fidelity, there is a peak in the curve of the FS as shown in Figs. 1(b) and 1(d). In Figs. 1(a) and 1(b), we can see that, as the parameter $M$ decreases, that is, as the anomalous dispersion strength of the medium decreases, the sudden decrease of the fidelity has a larger critical $z_{c}$ and correspondingly the peak on the curve of the FS also appears at a longer propagation distance. There is a similar effect when we change the frequency detuning $\Delta$. For a larger $\Delta$, which means a weaker anomalous dispersion near $\omega_{0}$, both the sudden decrease of the fidelity and the peak of the FS will appear at a longer propagation distance.

Figure 2 show the normalized temporal intensities in the moving time frame $\tau$ at different positions, which are denoted on the curve A of Fig. 1(d), as a smooth Gaussian pulse 
propagates through an anomalous dispersive medium. We can that the pulse almost keeps the same shape from Figs. 2(1) to 2(3) except for a small distortion. During this process, the FS is very small. As the propagation distance reaches the peak's position of the FS, the pulse's shape suffers a great change, and the intensity of the pulse's distortion has almost the same order with that of the pulse itself [see Fig. 2(4) to 2(5)]. After the FS's peak, the output pulse is totally distorted and has a completely different property from its initial pulse.

From a physical point of view, we know that the fidelity is a measurement of the overlap between the initial and output pulse spectral wavepackets. From the comparison between Figs. 1(a, c) and Figs. 1(b, d), we conclude that, when the fidelity decreases smoothly within a short propagation distance, the output pulse spectral wavepacket only suffers a quantitative deviation from its initial pulse spectral wavepacket. That is the output pulse is almost the same as the input one so that the FS changes very slow [almost a small constant before the peak of the FS, see in Figs. 1(b) and 1(d)]. However, when the fidelity has a sudden change at a critical distance $z_{c}$, actually there is a dramatic change in the output pulse spectral wavepacket. This tells us that the output pulse has a qualitative change from its initial pulse. Such a significant change in the fidelity always corresponds to a peak on the curve of the FS. Therefore, the position of the peak of the FS gives us the information of the maximal effective propagation distance before the output pulse suffers a qualitative change and becomes a completely distorted pulse.

From the information of Fig. 1 and Fig. 2, one can find that there is a maximal effective propagation distance for the propagation of a smooth Gaussian pulse in the double-gain abnormal dispersive medium. Within this maximal effective propagation distance, the shape of the output pulse could be seen as almost the same as that of the input one, otherwise the output pulse is completely distorted and has no longer any physical meaning for transferring the information of the original pulse. Here we would like to point out that the maximal propagation distance for a smooth Gaussian pulse depends on not only the dispersive strength of the medium but also the pulse spectral effective width.

In Fig. 3, we plot the changes of the fidelity and the FS for a smooth Gaussian pulse propagating through a double-absorptive normal dispersive medium with $M<0$ in Eq. (19). Similar to the cases in Figs. 1(a) and 1(c), one can find that the fidelity here is still a monotonical, decreasing function. However, differ from the cases in Fig. 1, there is no 
sudden change for the fidelity in Fig. 3(a), so that there is no peak on the curve of the FS in Fig. 3(b). Note that the value of the FS actually changes very small in this example [note the very small scale in Fig. 3(b)]. It means that there is no dramatic change (no qualitative change) for the output pulse when it propagates inside a normal dispersive medium. The inset figures in Fig. 3 show that the shape of the pulse is almost the same as the input one except for the pulse broaden effect. It tells us that the smooth pulse could always propagate effectively through a normal dispersive medium without losing its original shape.

Here we would like to point out that, from the above discussions, we can find that the group velocity is a good concept for the description of a smooth pulse's propagation inside the normal dispersive medium, while for the description of a smooth pulse's propagation inside the abnormal dispersive medium, the group velocity is only useful and effective within a certain propagation distance before the pulse suffers a significant change.

\section{B. A truncated Gaussian pulse}

Now let us turn to consider the propagation of a truncated Gaussian pulse passing through a dispersive media. Because for a real light pulse, people believe that there is always a start point and an end point. In our calculations, the initial wavepacket of a truncated Gaussian pulse in the time domain could be assumed to be

$$
\Psi\left(0, t_{1}\right)=\left\{\begin{array}{c}
A_{0} \exp \left[-\frac{t_{1}^{2}}{2 T_{0}^{2}}\right] \exp \left[-i \omega_{0} t_{1}\right],\left|t_{1}\right| \leq \xi \\
0, \quad \text { otherwise }
\end{array}\right.
$$

at the incident end $z=0$, where $\xi$ is a truncation parameter of the Gaussian pulse, here we always take $\xi>2 T_{0}$ for a weak truncation. Although the pulse's shape is similar to the previous case, there are two special points, a sudden switch-on point at $t_{1}=-\xi$ and a sudden switch-off point at $t_{1}=\xi$. In this situation, the corresponding pulse spectral wavepacket in the frequency domain could be numerically obtained by a Fourier transformation via Eq. (5). Using Eq. (41), we can also readily find the pulse spectral wavepacket at any position inside dispersive media. Similar to the above discussion of a smooth Gaussian pulse, we can also obtain the change of the fidelity $F(0, z)$ for a truncated Gaussian pulse. From the above discussions, since we have already known that a sudden change in the fidelity

always corresponds to a peak in the change of the FS, in the following discussion we only calculate the change of the FS for a truncated Gaussian pulse inside the dispersive medium 
and then we discuss the propagation properties of the output pulse. In the below, we still take $\omega_{0} / 2 \pi=3.5 \times 10^{14} \mathrm{~Hz}$, and pulse temporal width $T_{0}=1.2 \mu \mathrm{s}$.

In Fig. 4(a), we plot the changes of the FSs for different truncated Gaussian pulses propagating inside a double-absorptive normal dispersive medium with $M<0$ in Eq. (19)). From Fig. 4(a), we find that there exists a dramatic change in the FS curve for a truncated Gaussian pulse at a certain propagation distance. From the curves a to d, we find that the positions of the FS's peaks for different truncated pulses are strongly dependent on the truncation parameter $\xi$. For the small value of $\xi$ (i. e., the strong truncation on the pulse, see Eq. (22) ), it is found that the critical propagation distance (corresponding to the FS's peak) is very short, for example, see the curve a in Fig. 4(a). As the truncation of the pulse becomes weaker and weaker, i. e., the value of $\xi$ increases, it is seen that the peak of the FS appears at a longer propagation distance. Curve e displays the case for a smooth Gaussian pulse. By comparing the curves a, b, c, and d with the curve e, we find that for a smooth pulse inside the normal dispersive medium there is no qualitative change, while for a truncated pulse there is always a qualitative change and the position of such a FS's peak does strongly depend on the truncation parameter of the pulse. We suggest that such an essential difference between the smooth and the truncated pulses may be tested experimentally.

In order to understand such a dramatic change in the FS for a truncated Gaussian pluse inside the normal dispersive medium, we plot Fig. 4(b) to show the temporal normalized profiles at different propagation distances. Each figure corresponds to the position denoted on the curve b in Fig. 4(a). From Fig. 4(b) and the curve b in Fig. 4(a), we find that when the FS initially changes small, the pulse profile is almost preserved although it propagates through the normal dispersive medium with a slow group velocity. However, we notice that there is a sudden switch-on at the time $\tau=-\xi$ and a sudden switch-off at $\tau=\xi$ on the input pulse in the moving time frame; as the propagation distance increases, such small abrupt changes in the temporal pulse gradually dominates in the normalized profiles. From $z=0$ to $z=35 \mathrm{~cm}$ in Fig. 4(b), we see that the Gaussian-shaped peak actually propagates at the speed of the slow group velocity, while the sudden changes always occur at the moving times $\tau=-\xi$ and $\tau=\xi$ (i. e., at the speed of light in vacuum, see our definition of the moving time frame in section II). Near the position of the FS's peak, the pulse is mainly dominated by the distortion due to the sudden switch-on and -off. After the critical propagation distance, 
the pulse is completely dominated by the distortion (see the figure for the case of $z=50 \mathrm{~cm}$ ). Therefore the qualitative change for a truncated Gaussian pulse inside a normal dispersive medium comes from the strong distortion near the abrupt changes of the pulse profile.

Next we consider that such a truncated Gaussian pulse propagates through the above double-gain abnormal dispersive medium. Figure 5(a) shows the change of the FS under different truncation parameters. Similar to the cases in Fig. 4(a), the change of the FS inside the abnormal dispersive medium strongly depends on the value of the truncation parameter. For the strongly truncated Gaussian pulse, the position of the FS's peak occurs at a short propagation distance. For example, see the curve a for the pulse with $\xi=4 T_{0}$ in Fig. 5(a), the FS's peak is nearly located at $z=7.5 \mathrm{~cm}$; while for the curve $\mathrm{b}$ for the pulse with $\xi=5 T_{0}$, the peak is located near $z=10.5 \mathrm{~cm}$; the curve $\mathrm{d}$ is for the case with $\xi=12 T_{0}$, which corresponds to the case of a very weak truncated Gaussian pulse. For a comparison, we also plot the curve e for a smooth Gaussian pulse in such an abnormal dispersive medium. Clearly, the position of the FS's peak of the truncated Gaussian pulse is always smaller than that of a smooth Gaussian pulse inside the abnormal dispersive medium. This indicates that the effective propagation distance for a truncated Gaussian pulse is shorter than that for a smooth pulse; and it becomes shorter and shorter for a stronger truncation (i.e., a smaller $\xi)$.

Figure 5(b) plots the normalized pulse profiles at different positions on the curve b in Fig. 5(a). For a comparison, we use the dashed curve to denote the initial truncated Gaussian pulse, see Fig. 5(b). We find that the peak of the truncated pulse is advanced with the superluminal group velocity and the temporal shape is nearly preserved at these positions $\mathrm{z}=5 \mathrm{~cm}$ and $\mathrm{z}=7.5 \mathrm{~cm}$. Unlike the cases in Fig. 2, due to the effect of the sudden switch-on and -off of the truncated pulse, the pulse is gradually distorted at the beginning propagation region (within the effective propagation distance); then after the FS' peak, the pulse is completely distorted. This is why the truncated Gaussian pulse has a smaller effective propagation distance.

From the above discussion, in terms of the change of the FS for the truncated pulse, we therefore have that, inside the normal dispersive medium the group velocity is only valid before the FS's peak of a truncated Gaussian pulse happens, and inside the abnormal dispersive medium the effective propagation distance for the validity of the group velocity is much smaller than that of a smooth pulse. 


\section{SUMMARY}

In this work, we have used the concepts of the fidelity and the FS to describe the pulse propagation inside the dispersive media. We have found that both the fidelity and the FS are very useful for determining whether the pulse is completely distorted or not and confirming whether the group velocity of the pulse is physical meaningful or not. It has been found that there is a dramatic change in the changes of both the fidelity and the FS for a smooth Gaussian pulse propagating inside an abnormal dispersive medium, and the maximal effective propagation distance before the FS's peak is dependent on the dispersion strength of the medium, and after the FS's peak the pulse is completely distorted; however when a smooth Gaussian pulse propagates inside a normal dispersive media, there is no dramatic

change in the changes of the fidelity and its FS and therefore the pulse could always keep its original shape except for the pulse broaden effect. For a truncated Gaussian pulse, there always exists a qualitative change on the FS inside both the abnormal and normal dispersive media, and the maximal effective propagation distance in this case is strongly dependent on both the pulse itself (such as the truncation parameter) and the dispersive strength of the medium. We have found that, under the same medium's parameters, in terms of the position of the FS's peak, the effective propagation distance for a truncated pulse inside the dispersive medium is always much smaller than that of a smooth pulse. These results provide us a possible chance to analyze the pulse distortion and to determine the effective region of the pulse group-velocity description, and thus give us a deep understanding on the pulse propagation inside the dispersive media.

\section{Acknowledgments}

This work was supported by NSFC (No. 10604407) and CUHK 2060360.

[1] R. Y. Chiao, and A. M. Steinberg, in Progress in Optics, edited by E. Wolf (Elsevier, Amsterdam, 1997), vol. 37, p. 345; R. W. Boyd and D. J. Gauthier, in Progress in Optics, edited by E. Wolf, (Elsevier, Amsterdam, 2002), vol. 43, p. 497; P. W. Milloni, J. Phys. B: At. Mol. Phys. 35, R31 (2002). 
[2] M. M. Kash, V. A. Sautenkov, A. S. Zibrov, L. Hollberg, G. R. Welch, M. D. Lukin, Y. Rostovtsev, E. S. Fry, and M. O. Scully, Phys. Rev. Lett. 82, 5229 (1999); Q. Sun, Y. V. Rostovtsev, J. P. Dowling, M. O. Scully, M. S. Zubairy, Phys. Rev. A 72, 031802(R) (2005).

[3] S. Chu and S. Wong, Phys. Rev. Lett. 48, 738 (1982).

[4] L. J. Wang, A. Kuzmich, and A. Dogariu, Nature 406, 277 (2000).

[5] B. Pesala, Z. Chen, A. V. Uskov, and C. Chang-Hasnain, Opt. Exp. 14, 12968 (2006).

[6] K. J. Jiang, L. Deng, and M. G. Payne, Phys. Rev. A 76, 033819 (2007).

[7] K. Y. Song, K. S. Abedin, and K. Hotate, Opt. Exp. 16, 225 (2008).

[8] L. V. Hau, S. E. Harris, Z. Dutton, and C. H. Behroozi, Nature 397, 594 (1999).

[9] A. Kasapi, M. Jain, G. Y. Yin, and S. E. Harris, Phys. Rev. Lett. 74, 2447 (1995).

[10] M. Xiao, Y. Q. Li, S. Z. Jin, and J. Gea-Banacloche, Phys. Rev. Lett. 74, 666 (1995).

[11] M. A. Nilesen and I. L. Chuang, Quantum Computation and Quantum Information, (Cambrige University Press, Cambridge, England, 2000).

[12] For reviews, see A. Steane, Rep. Prog. Phys. 61, 117 (1998); D. P. DiVincenzo and C. H. Bennett, Nature (London) 404, 247 (2000).

[13] H. T. Quan, Z. Song, X. F. Liu, P. Zanardi, and C. P. Sun, Phys. Rev. Lett. 96, 140604 (2006).

[14] P. Zanardi and N. Paunkovic, Phys. Rev. E 74, 031123 (2006).

[15] M. Cozzini, P. Giorda, and P. Zanardi, Phys. Rev. B 75, 014439 (2007).

[16] P. Buonsante and A. Vezzani, Phys. Rev. Lett. 98, 110601 (2007).

[17] H. Q. Zhou and J. P. Barjaktarevic, J. Phys. A: Math. Theor. 41, 412001 (2008).

[18] S. J. Gu, Chin. Phys. Lett. 26, 026401 (2009).

[19] P. Zanardi, P. Giorda, and M. Cozzini, Phys. Rev. Lett. 99, 100603 (2007).

[20] W. L. You, Y. W. Li, and S. J. Gu, Phys. Rev. E 76, 022101 (2007).

[21] S. J. Gu, H. M. Kwok, W. Q. Ning, and H. Q. Lin, Phys. Rev. B 77, 245109 (2008).

[22] For a review, see S. J. Gu, arXiv: 0811.3127.

[23] L. G. Wang, N. H. Liu, Q. Lin, and S. Y. Zhu, Phys. Rev. E 68, 066606 (2003).

[24] S. Y. Zhu, Y. P. Yang, L. G. Wang, N. H. Liu, and M. S. Zubairy, arXiv: physics/0310026.

[25] K. Kim, H. S. Moon, C. Lee, S. K. Kim, and J. B. Kim, Phys. Rev. A 68, 013810 (2003).

[26] G. S. Agarwal, T. N. Dey, and S. Menon, Phys. Rev. A 64, 053809 (2001).

[27] M. Sahrai, H. Tajalli, K. T. Kapale, and M. S. Zubairy, Phys. Rev. A 70, 023813(2004). 
[28] M. Mahmoudi and J. Evers, Phys. Rev. A 74, 063827 (2006).

[29] L. G. Wang, S. Qamar, S. Y. Zhu, and M. S. Zubairy, Phys. Rev. A 77, 033833 (2008). 


\section{FIGURE CAPTIONS}

FIG. 1. Changes of the fidelity (a, c) and the FS (b, d) of a smooth Gaussian pulse inside an abnormal dispersive medium under different medium's parameters. In (a) and (b), different curves correspond to different $M$; and in (c) and (d), different curves correspond to different $\Delta$. Other parameters are $M / 2 \pi=22.62 \mathrm{~Hz}, \Delta / 2 \pi=1.35 \mathrm{MHz}, \gamma / 2 \pi=0.46 \mathrm{MHz}$, and the carried frequency $\omega_{0} / 2 \pi=3.5 \times 10^{8} \mathrm{MHz}$.

FIG. 2. Changes of the normalized intensities of smooth Gaussian pulses propagating through an abnormal dispersive medium at different positions on the curve A in Fig. 2(d).

FIG. 3. Changes of the fidelity (a) and the FS (b) of a smooth Gaussian pulse inside a normal dispersive medium. Inset figures show the normalized pulse profiles at different positions. Other parameters are the same as in Fig. 2 except for $M / 2 \pi=-22.62 \mathrm{~Hz}$.

FIG. 4. (a) Change of the FS for different truncated Gaussian pulses inside a normal dispersive medium with different truncated parameters: curve a for $\xi=4 T_{0}$, curve b for $\xi=5 T_{0}$, curve $\mathrm{c}$ for $\xi=6 T_{0}$, curve $\mathrm{d}$ for $\xi=7 T_{0}$, and cure e for the smooth Gaussian pulse. Figs. (1) to (6) show the nomalized intensities of the truncated Gaussian pulse at different positions on curve b in Fig. (a). The arrows in Figs. (2) and (3) indicate the sudden changes. The medium's parameters are the same as in Fig. 4.

FIG. 5. (a) Change of the FS for different truncated Gaussian pulses inside an abnormal dispersive medium with different truncated parameters: curve a for $\xi=4 T_{0}$, curve b for $\xi=5 T_{0}$, curve $\mathrm{c}$ for $\xi=7 T_{0}$, curve $\mathrm{d}$ for $\xi=12 T_{0}$, and curve e for the untruncated Gaussian pulse. Figs. (1) to (4) show the nomalized intensities of the truncated Gaussian pulse at different positions on curve b in Fig. (a). Te dashed curves in Figs. (1)-(4) denote the initial truncated Gaussian pulse. 
FIG. 1: FIG.1 
FIG. 2: FIG.2

FIG. 3: FIG.3 
FIG. 4: FIG.4 
FIG. 5: FIG.5 


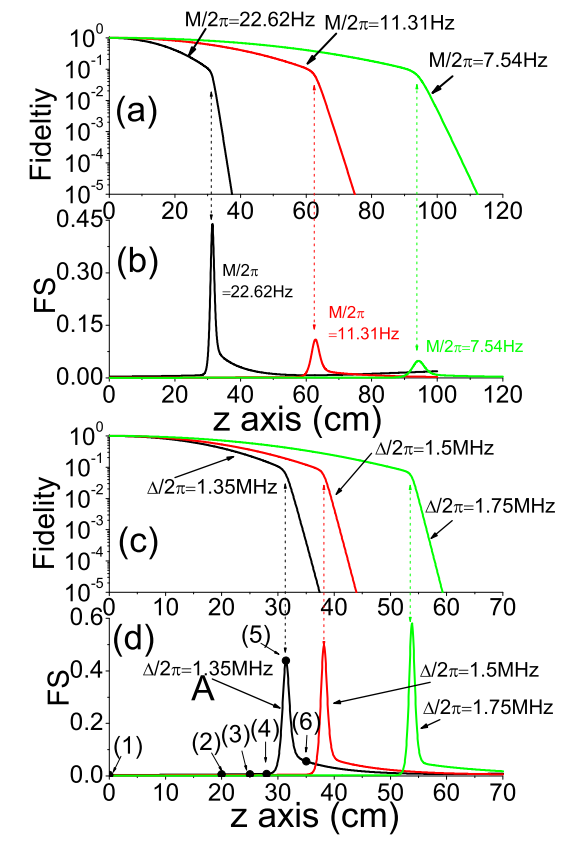




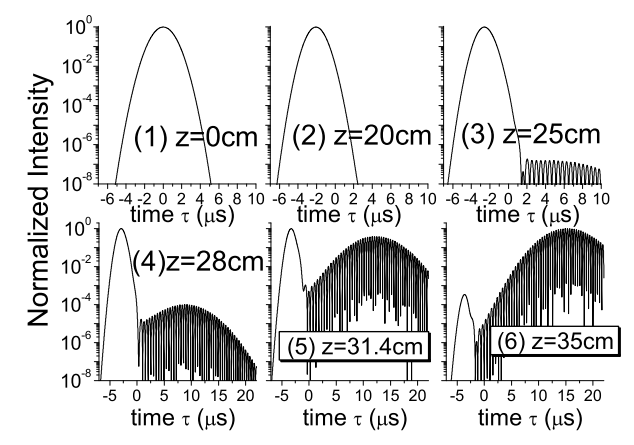




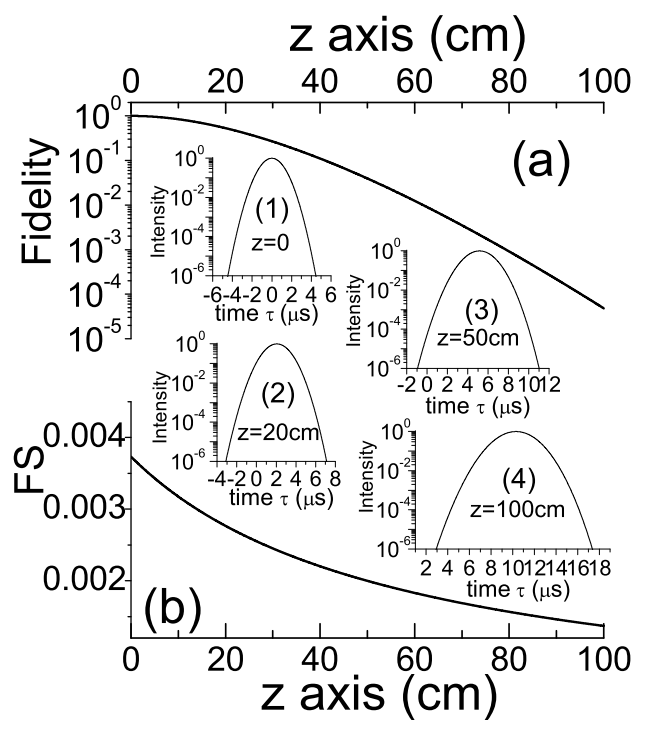




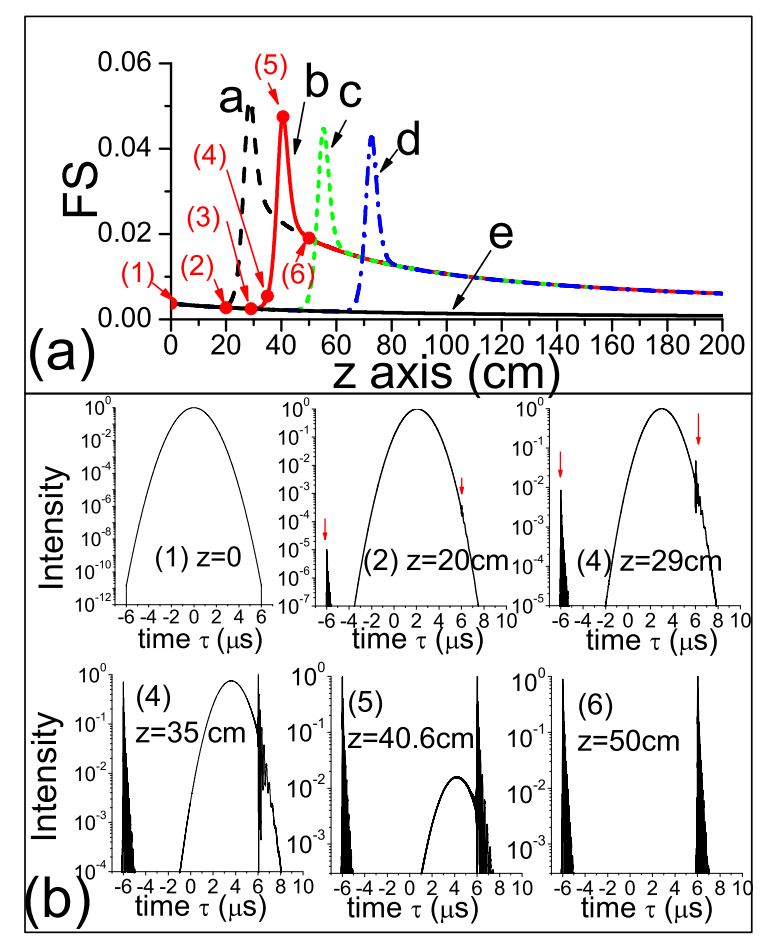




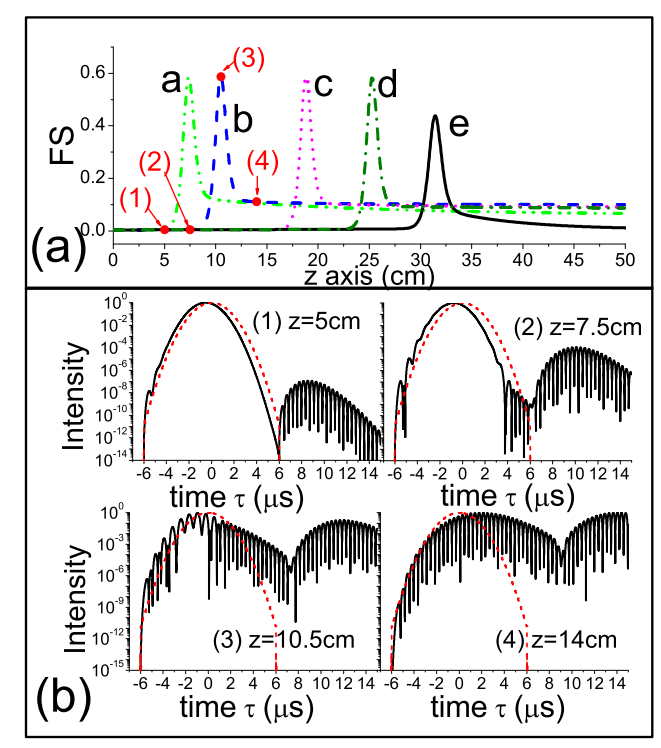

